\title{
Fabrication and testing of polymer-based capacitive micromachined ultrasound transducers for medical imaging
}

\author{
Carlos D. Gerardo ${ }^{1}$, Edmond Cretu ${ }^{1}$ and Robert Rohling ${ }^{1,2}$
}

\begin{abstract}
The ultrasonic transducer industry is dominated by piezoelectric materials. As an emerging alternative, capacitive micromachined ultrasound transducers (CMUTs) offer wider bandwidth, better integration with electronics, and ease of fabricating large arrays. CMUTs have a sealed cavity between a fixed electrode and a suspended metalized membrane. Manufacturing cost and sensitivity are limiting factors in current CMUTs that depend on the fabrication equipment and, especially, on the materials used. For widespread use of CMUTs, a much lower fabrication cost that uses inexpensive materials, which maintain or improve upon existing sensitivity, is needed. Herein, a new fabrication process is described for polymer-based CMUTs (polyCMUTs) using the photopolymer SU-8 and Omnicoat. The first ultrasound B-mode image of a wire phantom created with a 64-element linear array using synthetic aperture beamforming techniques is presented. $A 12 V_{A C}$ signal superimposed on a $10 V_{D C}$ signal was used on the transmission side, and only a bias-tee, with no amplifiers, was used on the receiving side. The low operational voltage and high sensitivity of this device can be partially attributed to a pre-biasing condition on the membrane. By using a novel sacrificial layer combined with a top electrode embedded inside the membrane, we demonstrated that SU-8 can be used to manufacture CMUTs inexpensively. Moreover, the fabrication used relatively simple equipment, and the number of fabrication steps was reduced compared to traditional CMUT fabrication. This new fabrication process has the potential to increase the use of CMUTs in the ultrasound market, including the market for wearable transducers.
\end{abstract}

\section{Introduction}

Ultrasound imaging is the most widely used medical imaging modality in the world when considering the number of images created annually. The forecasted growth for the ultrasound market from US $\$ 4.6$ billion in 2012 to almost US $\$ 7$ billion by 2019 (ref. 1) has boosted research in medical ultrasound fields, especially with regards to transducer design.

The ultrasonic transducer industry is dominated by piezoelectric materials, with incremental development of the same basic transduction mechanism detailed almost a

\footnotetext{
Correspondence: Carlos D. Gerardo (cgerardo@ece.ubc.ca)

${ }^{1}$ Department of Electrical and Computer Engineering, University of British Columbia, 2332 Main Mall, Vancouver, BC, Canada

${ }^{2}$ Department of Mechanical Engineering, University of British Columbia, 6250

Applied Science Ln, Vancouver, BC, Canada
}

century ago. An appropriate acoustic impedance matching between the transducer and the medium is paramount in ultrasound imaging systems, as this impedance matching has a profound impact on the efficiency of the system. For current piezoelectric-based systems, there is a high acoustic impedance mismatch between piezoelectric crystals ( 30 MRayl) and soft tissues ( 1.5 MRayl). Typically, layers of high-density rubber, which act as an acoustic matching layer, are placed between the transducer and the medium. Although piezoelectric imaging transducers having fractional bandwidths of the order of $80 \%$ and higher are becoming gradually available, the majority of the imaging transducers exhibit limited bandwidths, typically in the range $30-50 \%$ (ref. 2).

Capacitive micromachined ultrasound transducers (CMUTs) have emerged as alternatives to piezoelectric 
imaging transducers ${ }^{3}$. A CMUT is essentially a parallelplate capacitor with a fixed electrode at the bottom and a clamped metalized membrane suspended above a cavity. Ultrasound waves are generated when an AC signal, usually superimposed on a DC voltage, is applied between both electrodes; conversely, ultrasound waves can be detected by measuring the variation in capacitance of the device while a DC voltage is applied in the presence of incoming ultrasound waves.

CMUTs exhibit certain advantages over their piezoelectric counterparts, such as wider bandwidth, better integration with electronics, and ease of fabricating large arrays ${ }^{4}$.

The electromechanical coupling coefficient $k$ characterizes the efficiency of an ultrasound transducer. For piezoelectric transducers the bandwidth is determined by $k$ (ref. 5); while a high $k$ is desirable for the useful vibration mode, a low $k$ is often required for other competing modes, in order to suppress the spurious response caused by mode coupling ${ }^{6}$. This $k$ value is not constant and depends on the shape and dimensions of the transducer ${ }^{7}$; other factors such as backing substrate, residual stress, and electrode coverage can also affect this coefficient ${ }^{8}$. In CMUTs, a high $k$ leads to higher transducer sensitivity, improved bandwidth, and therefore improved image resolution'. Efficiency levels reported for CMUTs can be as high as $82 \%$ (ref. 10). The wide bandwidth for CMUTs is also the inherent result of the CMUT cell structure; the thin membrane clamped at the rim has a low mechanical impedance, which facilitates better acoustic matching to the medium ${ }^{11}$.

Conventional piezoelectric arrays are typically fabricated by mechanically dicing piezoelectric crystals and filling the kerfs with a polymer. Using this approach, the fabrication of ultrasonic arrays operating above $20 \mathrm{MHz}$ is very challenging ${ }^{12}$. This contrasts with the wide range of operating frequencies of CMUTs, some of them reportedly reaching $60 \mathrm{MHz}^{13}$.

In array operation, the parasitic capacitance of the interconnect between an element and its electronics is the limiting factor for the dynamic range and frequency bandwidth; therefore, it is preferred to have the electronics as close to the array elements as possible ${ }^{14}$. For piezoelectric two-dimensional (2D) arrays, a considerable sacrifice in the element area is required if an individual addressing is needed. The advantage of CMUTs in this respect is the fabrication of $2 \mathrm{D}$ arrays using through-wafer interconnections $^{14}$ or trench-isolated interconnects ${ }^{15}$, where individual CMUT cells can be electrically accessed from the back side of the substrate. Another alternative is to use low-temperature processes that allow the fabrication of CMUTs directly above pre-existing components such as amplifiers and beamformers ${ }^{16}$.

Despite the advantages offered by CMUTs over piezoelectric transducers, they also have some important drawbacks. CMUT transducers suffer from acoustic crosstalk that contributes to image degradation caused by Stoneley waves at the membrane-water interface and Lamb waves propagating in the substrate ${ }^{17}$. Current CMUT designs have limited receiving sensitivity, which thereby reduces the signal-to-noise ratio and penetration depth into tissues ${ }^{18}$; this has been tested experimentally $^{19,20}$, obtaining $10 \mathrm{~dB}$ less sensitivity for CMUT-based ultrasound probes compared to piezoelectric ones. Similar results regarding the penetration depth were obtained by Legros et al. ${ }^{21}$; nevertheless, the CMUTs outperformed piezoelectric probes in terms of the signal-to-noise ratio when phased-array steering was used; this was attributed to the larger acoustic radiation field experimentally measured from CMUTs.

A comprehensive and detailed description of the fabrication technologies for CMUTs is described by Ergury et $\mathrm{al}^{22}$. From this review, surface micromachining and wafer-bonding technologies emerge as the most important alternatives. In surface micromachining, the cavity underneath the membrane is created by depositing or growing a sacrificial layer on the carrier substrate. After membrane deposition, the sacrificial layer is removed with an etchant that is specifically chosen to dissolve the sacrificial material via etch channels without damaging the membrane material ${ }^{23}$. In the wafer-bonding method, the membrane and the cavity are defined on separate wafers that are bonded together under vacuum conditions ${ }^{24}$. Given that etching channels are not required, the fabrication process is simplified, and a higher fill factor can be achieved.

Silicon nitride and polysilicon are the most popular materials for fabricating CMUT membranes, while chromium and aluminum are typically used to pattern electrodes on top of these membranes. These materials are chosen mainly for their mechanical properties, such that the membranes can be as thin as possible, in order to minimize the effective gap between the bottom and top (or hot) electrodes. By decreasing the effective gap between electrodes, the electric field can be increased, and the impedance matching to the electronics can be improved $^{22}$. Once the desired operational frequency and the maximum biasing voltage have been identified, the CMUT membranes should be designed as thick as possible, given that their bandwidths linearly increase with thickness ${ }^{25}$.

Photopolymers are inexpensive, can be patterned using ultraviolet (UV) light, and have been used for the fabrication of microelectromechanical systems (MEMS) devi$\operatorname{ces}^{26,27}$. Their low density and high mechanical strength make them suitable for CMUTs because the impedance matching with the medium can be improved. Nonetheless, fabricating CMUTs using polymers is challenging because a thick membrane with a metal electrode on top 
is needed to reach the $\mathrm{MHz}$ region, contravening the required short gap between electrodes for low operational voltages and maximum sensitivity. Thus, despite some research in fabricating CMUTs using polymeric materi$\mathrm{als}^{28-30}$, the operational voltages were in the order of hundreds of volts, making them less attractive to be used in biomedical ultrasound industries ${ }^{31}$. Moreover, the mentioned devices were reported to be only operating in air.

Despite the advantages of polymers for fabricating CMUTs, the main limitation for their applications is the large effective gap between electrodes and the requirement to be properly sealed for operating in fluids. Our solution for this problem was to embed the electrode inside the membrane to bring it closer to the bottom electrode, without sacrificing the overall thickness required to be in the $\mathrm{MHz}$ range.

Herein, we present a new fabrication process for CMUTs using polymeric materials and demonstrate for the first time the use of an operational CMUT array in a liquid medium. By encapsulating the electrode inside the membrane, instead of being on top, polymer-based CMUTs (polyCMUTs) were able to obtain low operational voltages and frequencies that were comparable to traditional CMUTs fabricated in silicon nitride or polysilicon.

The total estimated amortized manufacturing cost for the prototype polyCMUT array presented is below US $\$ 100$. This is due to both the low cost of the materials used and the minimal manufacturing equipment required (mask aligner, metal evaporator, critical point dryer). Because the device is vacuum-sealed, the effective capacitance of the device is naturally increased by the atmospheric pressure as it deflects the membrane $27 \%$ of the total cavity height. Preliminary results indicate that these polyCMUTs can be pre-biased such that they can be operated even as passive devices (no external power) during reception and with low excitation voltages (10 $\mathrm{V}_{\mathrm{DC}}+12 \mathrm{~V}_{\mathrm{AC}}$ ) during transmission.

The materials and solvents used during manufacturing have low toxicity, and the sealing layer covering the device is considered to be biocompatible. The maximum process temperature used was $150^{\circ} \mathrm{C}$, meaning that polyCMUTs can be potentially fabricated directly on top of substrates containing pre-existing components, such as beamformers and $\mathrm{Tx} / \mathrm{Rx}$ switches. No acoustic matching layers were used. A novel sacrificial layer with high etching selectively was used, which can be spin or spray coated over rigid or flexible substrates, for wearable applications. The manufacturing process can be theoretically scaled up to roll-to-roll fabrication, decreasing the costs even further. This article is focused on the fabrication side of polyCMUTs that can be produced in a basic microfabrication facility.

\section{Materials and methods \\ Material selection}

In addition to improvements in the effective bandwidth, sensitivity, and radiated output pressure, a proper selection of fabrication materials can lead to improvements in the fabrication process and, in turn, lower the cost. We specifically selected the photopolymer SU-8 2000 series $^{32}$ as a structural material for our applications, given its unique dielectric and thermal properties, as well as its low density, photopatternability, optical transparency, and mechanical flexibility.

A crucial component in the fabrication of the cavities required for CMUTs is the sacrificial layer, where a high selectivity of the etchant is required to properly dissolve the sacrificial layer without damaging the membranes.

Different materials have been used as sacrificial layers when working with SU-8. Song et al. ${ }^{33}$ used overbaked positive photoresists to withstand the attack of solvents present in SU-8 to form electroplating molds, and the sacrificial layer was later removed using plasma etching. Moser et al. ${ }^{34}$ altered the chemical composition of SU-8 to make it more sensitive to UV light, and a standard SU-8 composition was used as a sacrificial layer to create microchannels. Foulds et $\mathrm{al}^{35}$ patterned polydimethylglutarimide (PMGI) to be used as a sacrificial layer, using a combination of positive photoresists and repetitive sequences of near UV and deep UV light exposures. Chiriaco et $\mathrm{al}^{36}$ created microchannels by patterning PMGI without using deep UV light; the masking material for PMGI was removed in acetone, leaving the sacrificial layer prone to damage since acetone also attacks the PMGI. Finally, different metals have also been used as sacrificial layers ${ }^{37,38}$, where they are either evaporated or sputtered, requiring long deposition times and leaving rough surfaces. Metals also require extra fabrication steps to be patterned for use as sacrificial layers.

One of the main novelties of this work is the use of a well-known material in a novel way to create the CMUT cavities. Omnicoat ${ }^{32}$ has been widely used as a lift-off material to release structures from silicon wafers. It has an excellent selectivity during etching and enhances the adhesion of photoresists to different substrates.

Despite these properties, Omnicoat is not photosensitive and the typical thickness during spin coating ranges from 5 to $15 \mathrm{~nm}$. Since a sacrificial layer in the order of hundreds of nanometers is needed, we evaporated $85 \%$ of the solvents present in Omnicoat. Thus, a much denser version of the same chemical was used for spin coating. Using this approach, we were able to increase the coating thickness from 15 to $300 \mathrm{~nm}$ in a single step at 1000 RPM. Multiple coatings could be stacked, or a higher percentage of the solvents could be evaporated if an even thicker sacrificial layer is desired. 


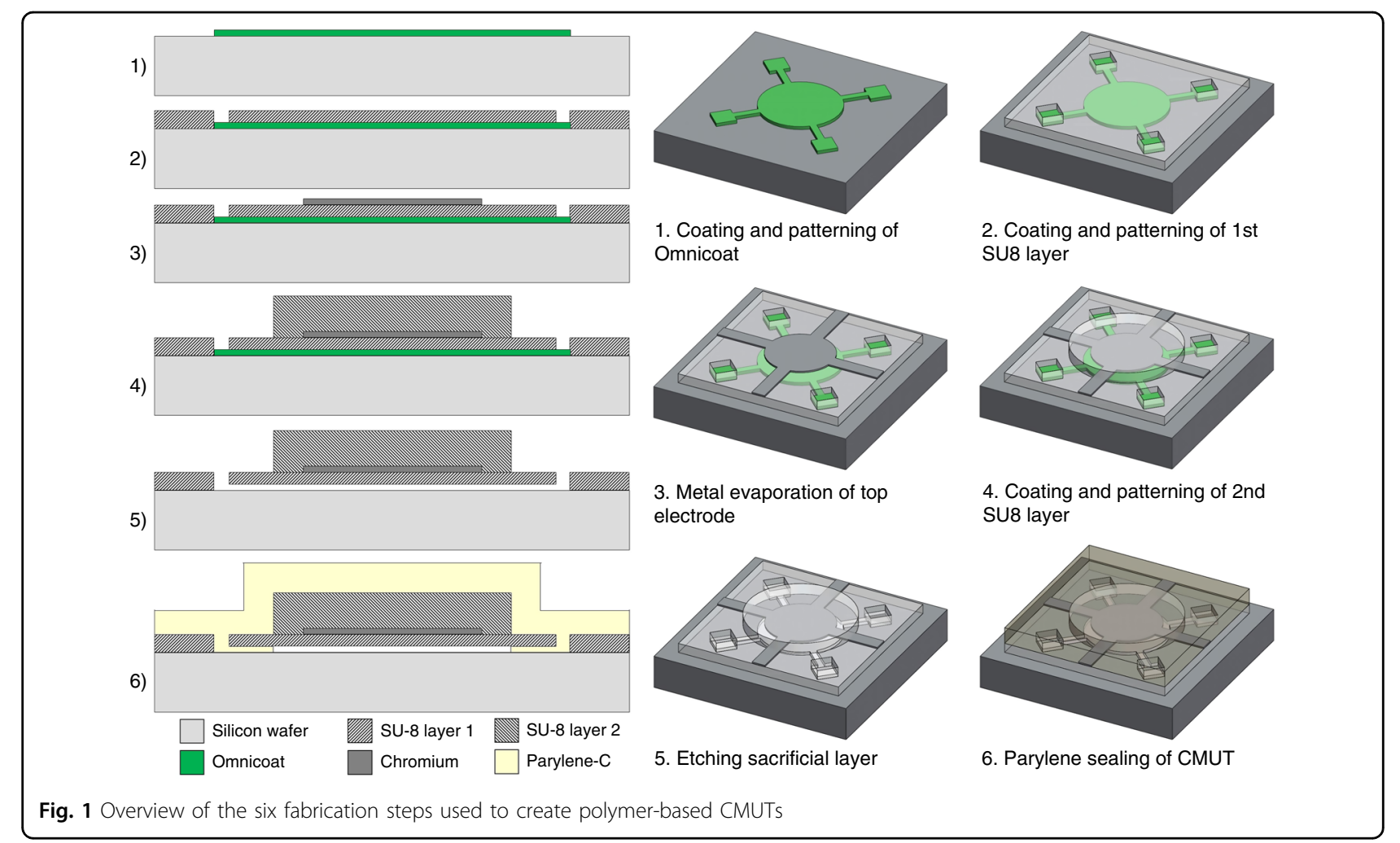

This might be useful in microfluidic applications where channels with different heights are typically used ${ }^{39}$. In our approach for CMUTs, the thickness of Omnicoat can be accurately controlled by spin coating, and it can be patterned at the same time as a masking material deposited on top. Omnicoat also has an excellent adhesion to different substrates, possesses excellent selectivity against solvents, and allows the substrates to be re-worked if necessary.

\section{Transducer fabrication}

Fig. 1 depicts the fabrication steps for these devices. A maskless lithographic system SF-100 (St. Petersburg, USA) was used for UV exposure given its rapid prototyping capabilities; however, if needed, a standard mask aligner with photomasks can be used to replicate the results presented in this article.

The fabrication started with a clean and low electrical resistance silicon wafer that acts as the bottom electrode. We spin coated the concentrated version of Omnicoat ( $15 \%$ of its original volume) onto the wafer and baked it at $150{ }^{\circ} \mathrm{C}$ for $3 \mathrm{~min}$ to obtain a $320 \mathrm{~nm}$ sacrificial layer. A layer of positive photoresist S1813 (ref. 32) was then deposited and patterned using UV light to create a masking layer to selectively remove the Omnicoat underneath. The sample was then immersed in alkalinebased MF319 developer ${ }^{32}$ for $60 \mathrm{~s}$, simultaneously etching the exposed areas of both S1813 and the Omnicoat; the etching was stopped by rinsing the sample in de-ionized water. The S1813 masking layer was then removed in acetone. Because of its excellent resistance to solvents, a patterned Omnicoat sacrificial layer was left intact without damage. A plasma etching step in $\mathrm{O}_{2}$ for $1 \mathrm{~min}$ is recommended at this time to completely eliminate all possible residues of Omnicoat left after etching and to increase adhesion of SU-8 to the silicon wafer. After plasma etching, the effective thickness of the sacrificial layer was $300 \mathrm{~nm}$.

A layer of SU-8 2000.5 was spun at 2000 RPM onto the sample to obtain a thickness of $0.67 \mu \mathrm{m}$. The thickness facilitated conformal coating of the Omnicoat areas that became the cavities and etch channels of our device. A short pre-baking step at $95^{\circ} \mathrm{C}$ was performed for $3 \mathrm{~min}$ prior UV exposure. The membranes, etching via holes, and clamping areas of the CMUTs were patterned in UV light, and the sample was post-exposure baked and developed. The SU-8 layer acts as a mechanical support for the top electrode and as dielectric material to avoid any short circuit since the dielectric strength of SU-8 reaches as much as $50 \%$ of that of $\mathrm{SiO}_{2}$ (ref. 40).

A layer of AZP 4110 photoresist was coated and patterned to act as a lift-off layer before evaporating on 570 $\mathrm{nm}$ of chromium. Chromium was selected because of its excellent adhesion properties to SU-8 films and low electrical resistivity ${ }^{41}$. A metal evaporation was preferred over sputtering because of its directional deposition, 
Table 1 Parameters of fabricated polymer CMUTs

\begin{tabular}{ll}
\hline Component & Dimension \\
\hline Membrane radius & $50 \mu \mathrm{m}$ \\
Sacrificial layer thickness & $0.30 \mu \mathrm{m}$ \\
Membrane SU-8 layer 1 thickness & $0.67 \mu \mathrm{m}$ \\
Top electrode thickness & $0.57 \mu \mathrm{m}$ \\
Membrane SU-8 layer 2 thickness & $2.40 \mu \mathrm{m}$ \\
Passivation layer thickness & $3.67 \mu \mathrm{m}$ \\
\hline
\end{tabular}

which simplifies the lift-off process. Once the electrodes are properly patterned, a second layer of SU-8 2002 was spin coated at $1000 \mathrm{RPM}$ to obtain a thickness of $2.40 \mu \mathrm{m}$, thereby covering the previous stack of Omnicoat, SU-8, and chromium layers. After pre-baking, the sample was exposed to UV light to pattern the top part of the CMUT membranes, leaving open areas for the etching holes. The sample was cured at $150{ }^{\circ} \mathrm{C}$ for $5 \mathrm{~min}$ and then gradually cooled down to room temperature. This annealing step removes any possible cracks created and increases the Young's modulus of the SU-8 films ${ }^{42}$.

The sample was then immersed in the developer MF319 (ref. 32). This etchant penetrates through the etch channels and gradually removes the Omnicoat material underneath the cavities. The circular cavities have a radius of $50 \mu \mathrm{m}$, and the etch channels are $5 \mu \mathrm{m}$ wide and $15 \mu \mathrm{m}$ long. After $3 \mathrm{~h}$, the Omnicoat sacrificial layer was completely removed. The sample was then immersed in deionized water for $2 \mathrm{~h}$ to displace the MF319 developer trapped inside the cavities. Finally, the sample was immersed in isopropanol to replace the water.

A Tousimis (Rockville, MD, USA) critical point dryer was used to completely release the membranes, avoiding any stiction problems ${ }^{43}$. In order to make our membranes watertight, a $3.67 \mu \mathrm{m}$ layer of Parylene- $\mathrm{C}$ was deposited in a low-pressure chamber, creating a sealed vacuum cavity inside the CMUT cell. In addition to providing excellent electrical insulation, Parylene- $\mathrm{C}$ is also biocompatible, optically transparent, has a low Young's modulus, and has a coefficient of water absorption close to zero ${ }^{44}$. The dimensions of the fabricated CMUTs are specified in Table 1. The materials used allow for a visual inspection of the fabricated device, facilitating the identification of any possible defect.

A slight modification in the process can lead to the fabrication of polyCMUTs on flexible substrates. Polyimide is a good candidate for this purpose given its elevated thermal stability, strong chemical resistance, and potential biocompatibility ${ }^{45,46}$; moreover, it has an excellent adhesion to metals and SU-8 films ${ }^{41,47,48}$. This polyimide film needs to be temporarily fixed to a rigid carrying substrate prior implementing the fabrication steps outlined in this article. This fabrication approach is still under investigation.

By using SU-8 and maskless lithography, a fully functional prototype was created in $17 \mathrm{~h}$. This compares favorable to multi-user wafer fabrication services, such as MEMSCAP $^{49}$ or MicraLyne ${ }^{50}$, where multiple designs are combined and processed with fixed fabrication protocols and long turnaround times. It is important to mention that the described fabrication process employs nonhazardous materials, that is, only organic solvents are used during manufacturing (acetone, isopropanol, SU-8 developer, and positive photoresist developer). The health risks associated with an accidental prolonged exposure to these materials do not go beyond drowsiness or minor skin irritation. This contrasts with the hazardous gas silane required to deposit silicon nitride and silicon dioxide in traditional CMUT fabrication ${ }^{51}$.

The final performance of the device is affected by several design parameters such as cavity height, membrane radius and thickness, electrode size, etc. The inherent resolution of the UV exposure system used allowed us to have release channels measuring $5 \mu \mathrm{m}$ in width and $15 \mu \mathrm{m}$ in length. Based on experimentation, a membrane radius of $50 \mu \mathrm{m}$ gave us an acceptable fill factor while maintaining a low risk of stiction during releasing.

The CMUT cavity was designed to account for the natural deflection of the membrane caused by the atmospheric pressure after vacuum sealing. The remaining gap should be large enough to allow the membrane to vibrate during normal operation given the maximum allowed voltage.

The thickness of the first layer of SU-8 depends on the maximum operating voltage of the CMUT; it should be as thin as possible to maintain a small effective gap between electrodes while providing a good electrical insulation to prevent any voltage breakdown in case the membrane is brought in contact with the bottom electrode. A thermal annealing of this layer removes any possible microcracks and enhances the adherence of SU-8 to the substrate and to the subsequent chromium layer. A gradual cooling procedure is recommended as it reduces the risks of delamination.

Chromium was chosen as the top electrode material based on the excellent adhesion strength to SU-8 (ref. 41) and to the fact that it requires one single masking layer during lift-off. The metallization radius was set to $46 \%$ of the membrane radius in order to increase the bandwidth of the final device ${ }^{25}$. This metal layer should be as thin as possible to minimize its effects on the mechanical properties of the membrane. A $500 \mathrm{~nm}$ layer was sufficient to maintain a good electrical conductivity between electrodes. Having a layer too thin can result in resistive interconnection paths that, combined with the capacitance of 


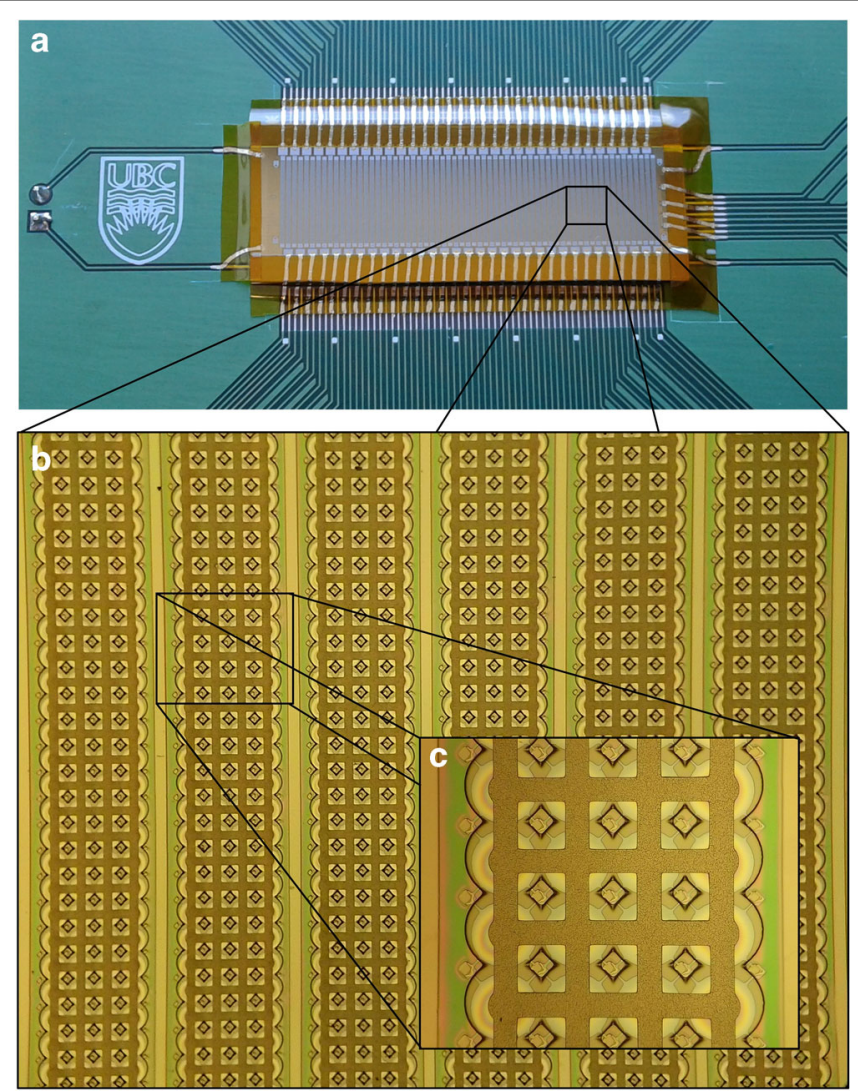

Fig. 2 a Sixty-four-element CMUT linear array mounted on PCB. b Six CMUT elements separated by a pitch of $550 \mu$ m. c CMUT cells showing interconnected top electrodes and cavities underneath, the optical transparency of SU-8 allows a visual identification of any possible defect

the CMUT cells, would behave as a natural low-pass RC filter that degrades the driving signals during excitation.

The thickness of the second SU-8 layer is tailored to the desired operational frequency of the device. Since the effective gap between the top and bottom electrodes (and therefore the operational voltage) is dictated by the cavity height and thickness of the first SU-8 layer, this design allows the fabrication of low-frequency and highfrequency CMUTs with constant operational voltages. This contrasts with the design in traditional CMUTs where the operational voltage increases with membrane thickness. The thickness of this second SU-8 layer should also take into account the effects from the Parylene-C layer during vacuum sealing as it has similar mechanical properties as SU-8.

\section{Results and discussions Optical characterization}

The final 64-element CMUT linear array was mounted and wired to a printed circuit board (PCB) interface circuit and is shown in Fig. 2. Each CMUT element contains $4 \times 75$ CMUT cells with a pitch of $550 \mu \mathrm{m}$ between elements. The top electrodes on each element are interconnected in parallel. We characterized the frequency response of the sealed device in air using a laser Doppler vibrometer OFV-5000 (Polytec, Irvine, CA, USA). The frequency response of the system and the first vibration mode are shown in Fig. 3a, yielding a quality factor of 40 in air. The topography of the final device was characterized using a white light interferometer (Polytec, Irvine, CA, USA) and is shown in Fig. 3b with the thick SU-8 membranes of the CMUT cells displayed in blue.

\section{Validation of operation}

The CMUT array was immersed in a tank filled with mineral oil to avoid any electrical short circuits between wirings. A curved C5-2 broadband piezoelectric ultrasound probe (BK Ultrasound, Richmond, CA, USA) was used as an acoustical source, using an aperture of 64 elements to send a focused ultrasound beam to the polyCMUT array placed $50 \mathrm{~mm}$ beneath. A $50 \mathrm{~V}$ square wave was applied to the piezoelectric transducer to generate a single focused ultrasound wave that arrives perpendicular to the CMUT membranes. The measured pulse on the CMUT is shown in Fig. 4 under different biasing conditions. An external bias of $10 \mathrm{~V}$ was applied through a bias-tee. The pre-bias condition is explained in the next section. 

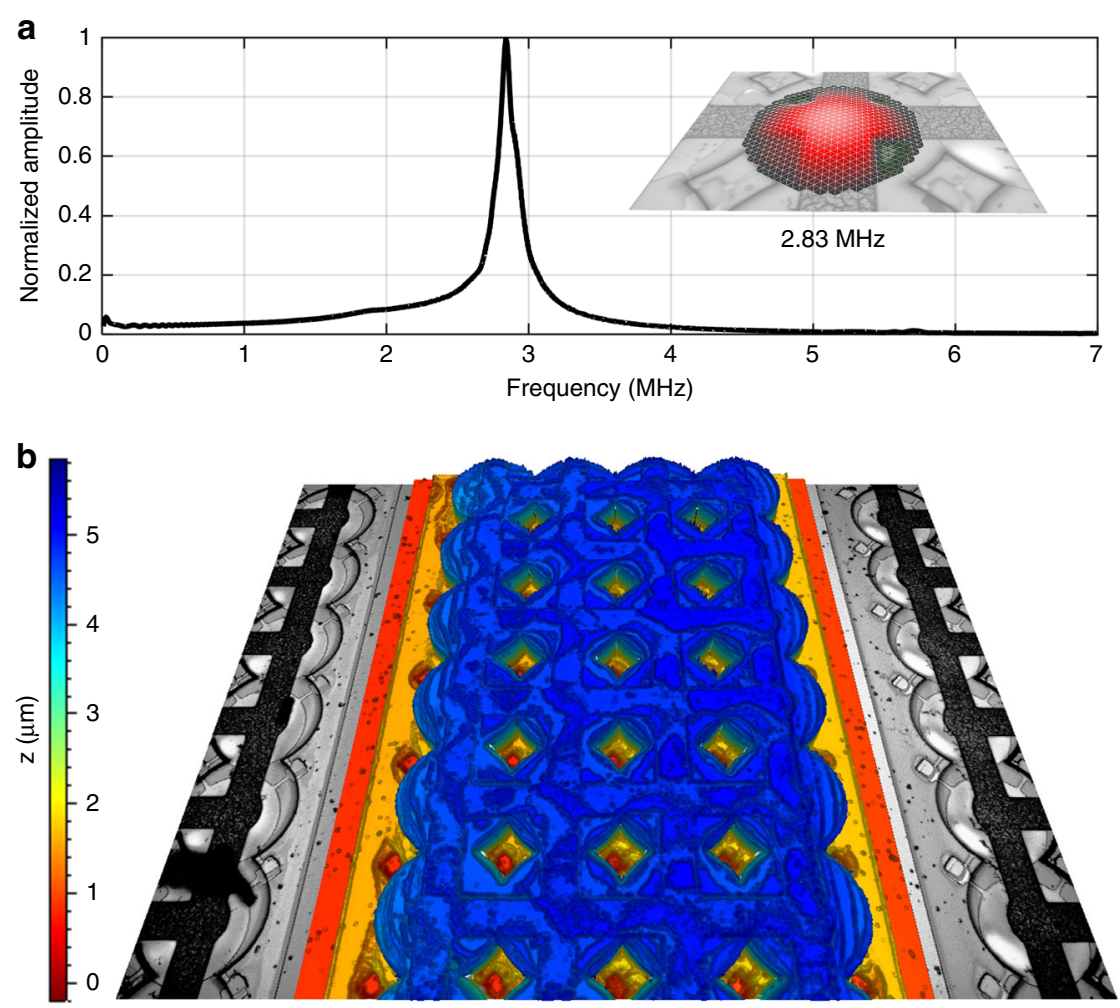

Fig. 3 a In air characterization of polymer CMUTs showing the first vibration mode at $2.83 \mathrm{MHz}$. b Static topography of a CMUT element using white light interferometry

The fast Fourier transform (FFT) of the measured pulse reveals that the $6 \mathrm{~dB}$ fractional bandwidth of this device is $101.6 \%$ around the center frequency of $1.525 \mathrm{MHz}$. This bandwidth for CMUTs is attributed to the relatively low fill factor of the design (59\%), given that each individual cell pushes the fluid sideways, as well as in the normal direction. As a result, the hydrodynamic mass of the fluid for each cell increases and, therefore, reduces the center frequency and bandwidth ${ }^{13}$.

An endurance test was performed in order to asses the durability of the fabricated device. A $2 \mathrm{MHz}$ sine wave superimposed on a $10 \mathrm{~V}_{\mathrm{DC}}$ was used to excite the polyCMUT linear array using a pulse repetition frequency of $5 \mathrm{kHz}$. The piezoelectric probe acted as receiver and recorded the detected pulses every $30 \mathrm{~min}$ for a period of $12 \mathrm{~h}$ of continuous operation, the results are shown in Fig. 5. Even though the polymer CMUT array underwent over 200 million excitation cycles, the amplitude of the signals stayed within $95 \%$ of the maximum detected peak. Further endurance tests involving high temperatures and elevated voltages are still under way.

\section{Pre-biasing}

Charge trapping in CMUTs occurs when electrical charges are trapped in insulating layers in a CMUT membrane. This phenomenon was initially considered as a negative effect in CMUTs because it shifts the resonant frequency of the membrane over time ${ }^{52}$. However, Park et al. $^{53}$ demonstrated that these electrical charges in CMUTs can be introduced in a well-controlled manner and used to operate a CMUT without any DC bias applied. The main benefit of having electrical charges trapped in the CMUTs is that they act as a built-in bias voltage, which suggests that only a small external DC voltage (compared to the required voltage if no trapped charges were present) is required to bring the membrane closer to the bottom electrode, that is, the effective gap between electrodes is reduced, thereby increasing its capacitance. As this effective capacitance is increased, a small perturbation to the membrane deflection caused by external acoustic sources will result in a large variation in the capacitance.

Direct detection, without amplification of ultrasound waves coming from the same piezoelectric transducer described in the previous section, was possible owing to this pre-biasing condition. Even though no external voltage is applied between the CMUT terminals, a $260 \mathrm{mV}_{\mathrm{PP}}$ echo signal was detected, as shown in Fig. 4.

The first SU-8 layer described in the fabrication steps has a dual purpose. First, it serves as an insulation layer between the top and bottom electrode, preventing a short circuit in case the membrane is brought in contact with 


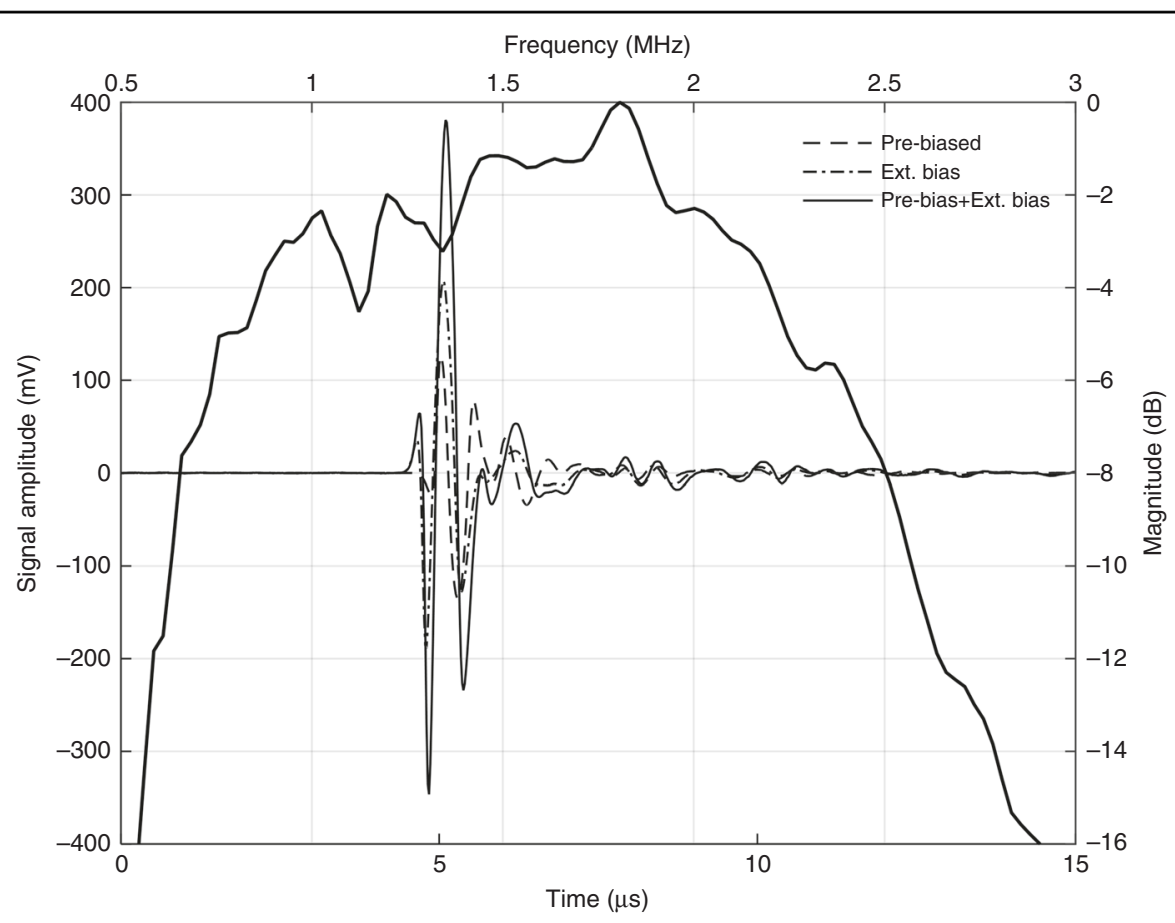

Fig. 4 Acoustic response in mineral oil from polymer-based CMUT and FFT of received acoustic pulse showing a 101.6\% fractional bandwidth

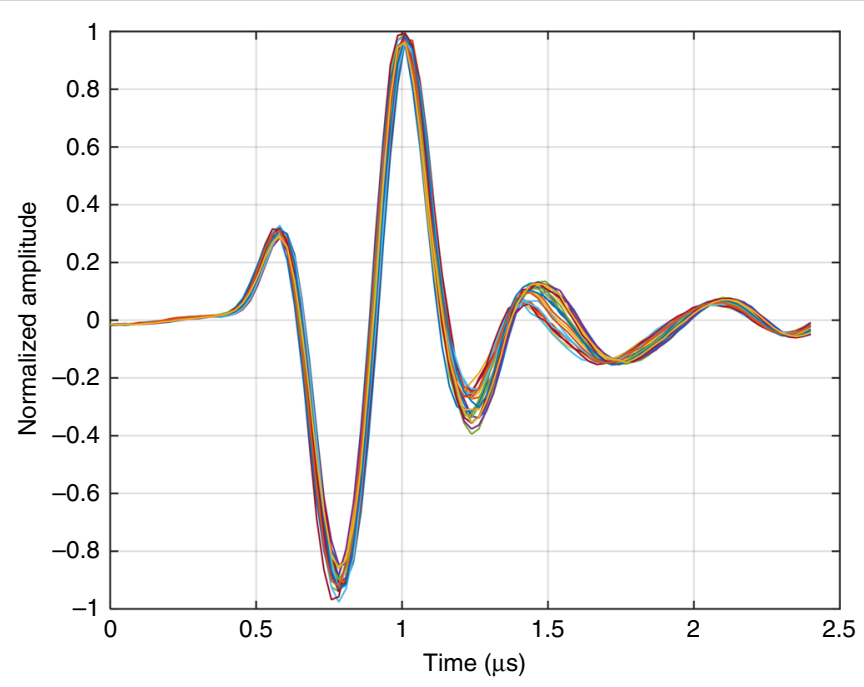

Fig. $\mathbf{5}$ Ultrasound pulses generated by polymer CMUTs recorded every $\mathbf{3 0} \mathbf{m i n}$ for a period of $\mathbf{1 2} \mathbf{h}$. Signals stayed within $95 \%$ of the maximum detected peak

the top electrode. Second, the SU-8 layer acts as a novel charge trapping layer. For the first time, SU-8 was used to retain electrical charges, acting as a built-in bias voltage.

As determined by experimentation, electrical charges are trapped in the CMUT membrane and create an intrinsic (built-in) bias voltage. This contributes to the natural deflection of the CMUT membrane and, therefore, lowers the external DC bias voltage required for actuation and reception. Following a similar procedure as used by Park et al. ${ }^{53}$, the CMUTs were "charged" by applying a DC voltage of $100 \mathrm{~V}$ between the top and bottom electrodes for $5 \mathrm{~min}$. During this time, the membrane collapsed against the bottom electrode, generating an electric field strength of $1.49 \mathrm{MV} / \mathrm{cm}$. After 


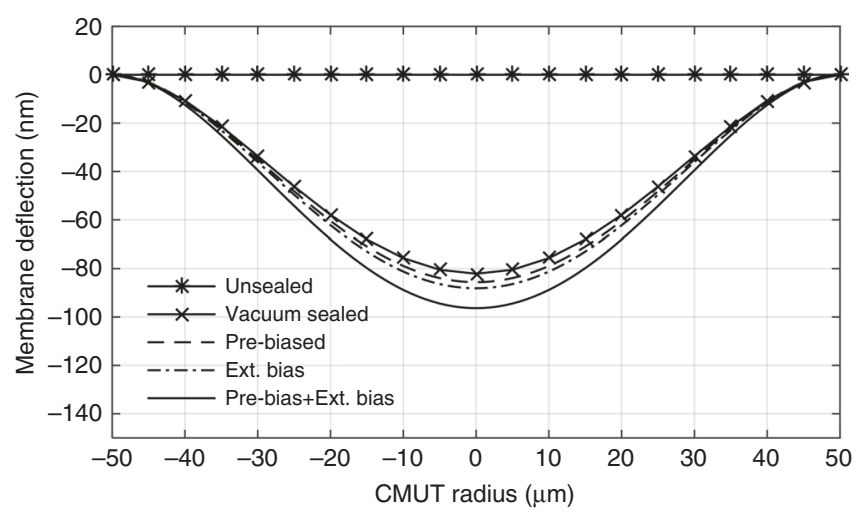

Fig. 6 Contribution of different factors towards the total CMUT membrane deflection

removing the bias voltage, the CMUT was connected to an impedance analyzer to obtain a $C-V$ curve. The symmetry point of this $C-V$ curve revealed that an intrinsic (built-in) voltage of $8.9 \mathrm{~V}$ was present. From this, an average trapped charge of $7.25 \times 10^{-8} \mathrm{C} / \mathrm{cm}^{2}$ was calculated. Larger intrinsic bias voltage could be experimentally obtained by charging the CMUTs for longer periods of time, for example, charging the CMUT for 210 min leads to a $30 \mathrm{~V}$ pre-bias condition.

The SU-8 membrane is naturally deflected towards the bottom electrode as a result of the atmospheric pressure. This serves to bring the top and bottom electrodes closer to each other, thereby increasing the capacitance. As the membrane becomes increasingly thin, this effect is increased $^{54}$.

Figure 6 shows the contribution of different effects towards the total deflection of the membrane. Atmospheric pressure is a major influence. Naturally developed by sealing the cavity in a vacuum chamber, atmospheric pressure deflects the membrane $82 \mathrm{~nm}$ (27\% of cavity height) towards the bottom electrode. The pre-biasing condition (built-in bias voltage) increases the deflection by $1.5 \%$. Lastly, the contribution of the external bias voltage increases the total membrane deflection to a maximum of $32.1 \%$ of the cavity height.

By embedding the electrode inside the membrane, we were able to obtain a pull-in voltage of $65 \mathrm{~V}$, which contrasts with the $220 \mathrm{~V}$ required when the metal electrode was placed directly above the CMUT membrane as in traditional CMUT designs.

\section{B-mode imaging}

The feasibility of using these polyCMUTs for the creation of B-mode images was assessed. An array of 12 aluminum wires with a diameter of $600 \mu \mathrm{m}$ equally spaced by $5 \mathrm{~mm}$ was used as a target in a tank filled with mineral oil as shown in Fig. 7a, b. The linear CMUT array was placed directly above the wires and excited by a $12 \mathrm{~V}_{\mathrm{PP}}$ square pulse that was oscillated at $2 \mathrm{MHz}$ and superimposed on a $10 \mathrm{~V}_{\mathrm{DC}}$ bias using a bias-tee. This external bias voltage was selected in order to avoid pull-in in case the CMUT was charged for a long period of time.

Only one CMUT element was used during transmit, thereby creating a quasi-cylindrical wavefront. The echoes created by the aluminum wires were captured by all other elements. For receiving, a $10 \mathrm{~V}_{\mathrm{DC}}$ bias was applied to the CMUT using a bias-tee. The echoes were measured by a digital oscilloscope, and averaged over 256 times to reduce electrical noise.

The received signals were later processed in MatLab (Natick, MA, USA) using synthetic aperture beamforming techniques $^{55}$. A simple Hanning window was applied as apodization and no time-gain compensation processing was used. A B-mode image generated using polyCMUTs is shown in Fig. 7c. Despite the unamplified measurement scheme, all of the 12 wires in the phantom can be identified to a depth of $85 \mathrm{~mm}$. The signal profiles of the first 5 wires of the phantom were taken in order to assess the lateral resolution of the polyCMUT array as shown in Fig. $7 \mathrm{~d}$, the full-width at half-maximum revealed a resolution of $<1.5 \mathrm{~mm}$.

\section{Conclusions}

This work provides a proof of concept that polymeric materials can be used to fabricate CMUTs for biomedical imaging. Having the top electrode embedded inside the membrane led to low operational voltages comparable to traditional CMUTs fabricated in polysilicon or silicon nitride. A 64-element CMUT linear array was successfully operated and characterized, leading to the first demonstration of a B-mode image created with polyCMUTs.

An advantage of using Omnicoat as sacrificial material is that the deposition method is simple and controllable. The thickness of this layer can be reduced to a few tens of nanometers if desired, leading to potentially increased sensitivity and even lower operational voltages. This is the 

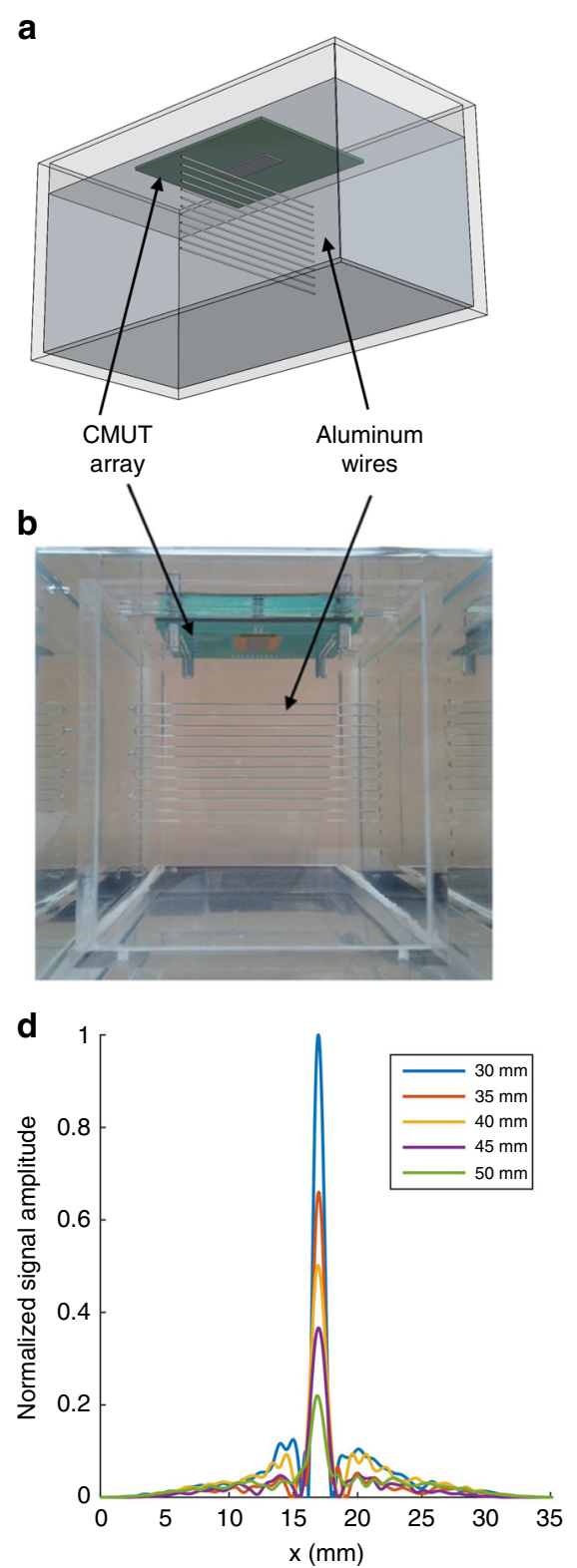
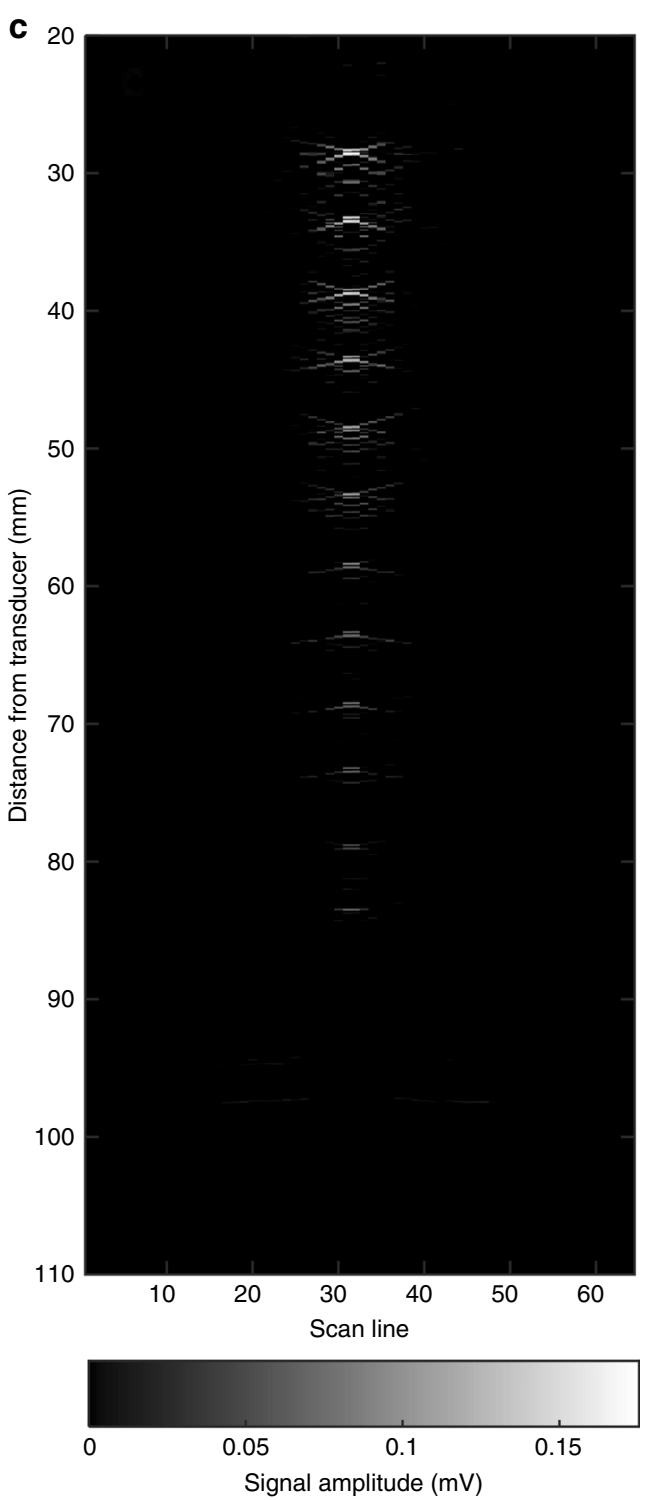

Fig. 7 a Diagram of the wire phantom used; 12 aluminum wires spaced by $5 \mathrm{~mm}$. b Photograph of the actual wire phantom immersed in mineral oil. c B-mode image of a wire phantom using a linear array of polymer-based CMUTs using a display dynamic range of $30 \mathrm{~dB}$. $\mathbf{d}$ Assessment of the lateral resolution, the FWHM for the first 5 wires down to a depth of $50 \mathrm{~mm}$ was $<1.5 \mathrm{~mm}$

first demonstration where a condensed version of Omnicoat is selectively patterned and used as sacrificial layer for SU-8.

The materials used were selected in such a way as to manufacture CMUTs inexpensively, minimize the fabrication steps, and obtain similar dimensions comparable to traditional CMUTs ${ }^{22}$. This work highlights the benefits of using a simple fabrication process with inexpensive materials coupled to basic microfabrication equipment. Although SU-8 photopolymer was used for this study, any other polymer or soft material can in principle be used for the fabrication of CMUTs. The important factor is to maintain a small effective gap between the electrodes of CMUTs for low operational voltages and high sensitivity.

A full acoustic characterization for the presented polyCMUTs is yet to be done. Performance tests for these devices are under way to create a pre-biasing mathematical model.

This technology has the potential to be extended to flexible substrates intended for conformal and wearable health monitoring systems ${ }^{56,57}$. This contrasts with traditional fabrication approaches that use a combination of 
semiconductors and metals as structural materials. Although flexible CMUT arrays have been derived from rigid substrates ${ }^{58,59}$, problems have been reported with electrical interconnections of individual elements getting cracked.

An advantage of this design is that the low-temperature process $\left(\max 150^{\circ} \mathrm{C}\right)$ can be seamlessly integrated with CMOS-compatible processes for closer integration with electronics. SU-8 can also be easily coupled with other materials, such as polydimethylsiloxane or polymethylmethacrylate, for microfluidics applications ${ }^{60,61}$.

A variation of the proposed fabrication methodology is to create the same version of polyCMUTs using waferbonding technologies for SU-8, as reported by Joseph et al. ${ }^{62}$. The fabrication methodology can also potentially be extended to roll-to-roll technology ${ }^{63}$, where CMUT cavities and membranes are defined on separate flexible substrates and then bonded together under a vacuum environment. This could decrease production times and costs even further, with the possibility of fabricating ultralow-cost ultrasound transducers.

\section{Acknowledgements}

We thank CMC Microsystems Canada and the Natural Sciences and Engineering Research Council of Canada (NSERC) for their funding, software, and fabrication support. We also thank Manyou Ma for her support with the synthetic aperture beamforming algorithm.

\section{Conflict of interest}

The authors declare that they have no conflict of interest.

Received: 17 January 2018 Revised: 4 April 2018 Accepted: 14 May 2018 Published online: 27 August 2018

\section{References}

1. Global Business Intelligence. Ultrasound Systems Market to 2019-Technological Advancements, Wide Applications and Device Portability to Drive Future Growth. (2013). Available at: http://www.gbiresearch.com/report-store/ market-reports/medtech/ultrasound-systems-market-to-2019-technologicaladvancements-wide-applications-and-device-portability-to-drive-futuregrowth.

2. Trots, I., Nowicki, A., Lewandowski, M., Secomski, W. \& Litniewski, J. The influence of the transducer bandwidth and double pulse transmission on the encoded imaging ultrasound. Hydroacoustics 11, 419-430 (2008).

3. Lawrence, W. C. \& Yeow, J. in Medical Imaging: Technology and Applications (eds Troy Farncombe and Krzysztof Iniewski) 253-270 (CRC Press, Boca Raton, $\mathrm{FL}, 2013)$.

4. Oralkan, O. et al. Capacitive micromachined ultrasonic transducers: nextgeneration arrays for acoustic imaging? Ultrason. Ferroelectr. Freq. Control IEEE Trans. 49, 1596-1610 (2002).

5. Yaralioglu, G. G., Ergun, A. S., Bayram, B., Haeggstrom, E. \& Khuri-Yakub, B. T. Calculation and measurement of electromechanical coupling coefficient of capacitive micromachined ultrasonic transducers. Ultrason. Ferroelectr. Freq. Control IEEE Trans. 50, 449-456 (2003).

6. Gallego-Juarez, J. A. Piezoelectric ceramics and ultrasonic transducers. J. Phys. E 22, 804 (1989).

7. Zhou, Q., Lam, K. H., Zheng, H., Qiu, W. \& Shung, K. K. Piezoelectric single crystals for ultrasonic transducers in biomedical applications. Prog. Mater. Sci. 66, 87-111 (2014).
8. Cho, J., Anderson, M., Richards, R., Bahr, D. \& Richards, C. Optimization of electromechanical coupling for a thin-film PZT membrane: I. Modeling. J. Micromech. Microeng. 15, 1797 (2005).

9. Ergun, A. S., Yaralioglu, G. G. \& Khuri-Yakub, B. T. Capacitive micromachined ultrasonic transducers: theory and technology. J. Aerosp. Eng. 16, 76-84 (2003).

10. Guldiken, R. O., Zahorian, J., Yamaner, F. \& Degertekin, F. Dual-electrode CMUT with non-uniform membranes for high electromechanical coupling coefficient and high bandwidth operation. Ultrason. Ferroelectr. Freq. Control IEEE Trans. 56, 1270-1276 (2009).

11. Park, K. K., Kupnik, M., Lee, H. J., Khuri-Yakub, B. T. \& Wygant, I. O. Modeling and measuring the effects of mutual impedance on multi-cell CMUT configurations. Ultrason. Symp. IUS IEEE 2010, 431-434 (2010).

12. Ritter, T. A., Shrout, T. R., Tutwiler, R. \& Shung, K. K. A 30-MHz piezo-composite ultrasound array for medical imaging applications. IEEE Trans. Ultrason. Ferroelectr. Freq. Control 49, 217-230 (2002).

13. Oralkan, O. et al. High-frequency CMUT arrays for high-resolution medical imaging. In IEEE Ultrasonics Symposium, 1, 399-402 (Montreal, QC, 2004). https://doi.org/10.1109/ULTSYM.2004.1417747.

14. Cheng, C.-H., Ergun, A. \& Khuri-Yakub, B. T. Electrical through-wafer interconnects with sub-picofarad parasitic capacitance. In Microelectromechanical Systems Conference, 18-21 (Berkeley, CA, 2001). https://doi.org/10.1109/ MEMSC.2001.992732.

15. Zhuang, X. et al. Through-wafer trench-isolated electrical interconnects for CMUT arrays. In IEEE International Ultrasonics Symposium (Rotterdam, Netherlands, 2005). https://doi.org/10.1109/ULTSYM.2005.1602894.

16. Daft, C. et al. Microfabricated ultrasonic transducers monolithically integrated with high voltage electronics. In 2004 IEEE Ultrasonics Symposium, 1, 493-496 (Montreal, QC, 2004). https://doi.org/10.1109/ULTSYM.2004.1417770.

17. Zhou, S. \& Hossack, J. A. Reducing inter-element acoustic crosstalk in capacitive micromachined ultrasound transducers. Ultrason. Ferroelectr. Freq. Control IEEE Trans. 54, 1217-1228 (2007).

18. Salim, M. S., Abd Malek, M. F., Heng, R. B. W., Juni, K. M. \& Sabri, N. Capacitive micromachined ultrasonic transducers: technology and application. J. Med. Ultrasound 20, 8-31 (2012).

19. Caronti, A. et al. Capacitive micromachined ultrasonic transducer (CMUT) arrays for medical imaging. Microelectron. J. 37, 770-777 (2006).

20. Mills, D. M. \& Smith, L. S. Real-time in-vivo imaging with capacitive micromachined ultrasound transducer (CMUT) linear arrays. In IEEE Ultrasonics Symposium 2003, 568-571 (IEEE, Honolulu, HI, 2003). https://doi.org/10.1109/ ULTSYM.2003.1293467.

21. Legros, M., Meynier, C., Dufait, R., Ferin, G. \& Tranquart, F. Piezocomposite and CMUT arrays assessment through in vitro imaging performances. In IEEE Ultrasonics Symposium, 1142-1145 (Beijing, China, 2008). https://doi.org/ 10.1109/ULTSYM.2008.0275.

22. Erguri, A. S. et al. Capacitive micromachined ultrasonic transducers: fabrication technology. Ultrason. Ferroelectr. Frea. Control IEEE Trans. 52, 2242-2258 (2005).

23. Ladabaum, I., Jin, X. C., Soh, H. T., Atalar, A. \& Khuri-Yakub, B. T. Surface micromachined capacitive ultrasonic transducers. IEEE Trans. Ultrason. Ferroelectr. Frea. Control 45, 678-690 (1998).

24. Huang, Yongli et al. Fabricating capacitive micromachined ultrasonic transducers with wafer-bonding technology. J. Microelectromech. Syst. 12, 128-137 (2003).

25. Bozkurt, A., Ladabaum, I., Atalar, A. \& Khuri-Yakub, B. T. Theory and analysis of electrode size optimization for capacitive microfabricated ultrasonic transducers. IEEE Trans. Ultrason. Ferroelectr. Freq. Control 46, 1364-1374 (1999).

26. Lorenz, H. et al. SU-8: a low-cost negative resist for MEMS. J. Micromech. Microeng. 7, 121 (1997).

27. Liu, C. Recent developments in polymer MEMS. Adv. Mater. 19, 3783-3790 (2007).

28. Li, Y. P. et al. Design and analysis of capacitive micromachined ultrasonic transducers based on SU-8. Key Eng. Mater. 645-646, 577-582 (2015).

29. Joseph, J., Singh, S. G. \& Vanjari, S. R. K. Fabrication of SU-8 based capacitive micromachined ultrasonic transducer for low frequency therapeutic applications. In 2015 International Conference of the IEEE Engineering in Medicine and Biology Society, 1365-1368 (Milan, Italy). https://doi.org/10.1109/ EMBC.2015.7318622

30. Chiou, D.-Y., Chen, M.-Y., Chang, M.-W. \& Deng, H.-C. Finite element modeling, characterization, and optimization design for the polymer-typed capacitive micro-arrayed ultrasonic transducer. Microsyst. Technol. 14, 787-797 (2008).

31. Dalziel, C. F. Electric shock hazard. IEEE Spectr. 9, 41-50 (1972). 
32. MicroChem: Innovative Chemical Solutions for MEMS and Microelectronics. Available at: http://www.microchem.com/. Accessed 4 Sept 2017.

33. Song, I. \& Ajmera, P. K. Use of a photoresist sacrificial layer with SU-8 electroplating mould in MEMS fabrication. J. Micromech. Microeng. 13, 816 (2003).

34. Moser, Y., Forti, R., Jiguet, S., Lehnert, T. \& Gijs, M. A. M. Suspended SU-8 structures for monolithic microfluidic channels. Microfluid. Nanofluid. 10 219-224 (2011).

35. Foulds, I. G., Johnstone, R. W. \& Parameswaran, M. Polydimethylglutarimide (PMGI) as a sacrificial material for SU-8 surface-micromachining. J. Micromech. Microeng. 18, 075011 (2008).

36. Chiriacò, M. S. et al. Fabrication of interconnected multilevel channels in a monolithic SU-8 structure using a LOR sacrificial layer. Microelectron. Eng. 164, 30-35 (2016).

37. Seidemann, V., Rabe, J., Feldmann, M. \& Büttgenbach, S. SU8-micromechanical structures with in situ fabricated movable parts. Microsyst. Technol. 8, 348-350 (2002).

38. Psoma, S. D. \& Jenkins, D. W. Comparative assessment of different sacrificial materials for releasing SU-8 structures. Rev. Adv. Mater. Sci. 10, 149-155 (2005).

39. Peeni, B. A., Lee, M. L., Hawkins, A. R. \& Woolley, A. T. Sacrificial layer microfluidic device fabrication methods. Electrophoresis 27, 4888-4895 (2006).

40. Melai, J., Salm, C., Smits, S., Visschers, J. \& Schmitz, J. The electrical conduction and dielectric strength of SU-8. J. Micromech. Microeng. 19, 065012 (2009).

41. Dai, W., Lian, K. \& Wang, W. A quantitative study on the adhesion property of cured SU-8 on various metallic surfaces. Microsyst. Technol. 11, 526-534 (2005).

42. Feng, R. \& Farris, R. J. Influence of processing conditions on the thermal and mechanical properties of SU8 negative photoresist coatings. J. Micromech. Microeng. 13, 80 (2003).

43. Tas, N., Sonnenberg, T., Jansen, H., Legtenberg, R. \& Elwenspoek, M. Stiction in surface micromachining. J. Micromech. Microeng. 6, 385 (1996).

44. Jeanne, E. et al. Evaluation of Parylene as protection layer for capacitive micromachined ultrasonic transducers. ECS Trans. 11, 25-33 (2008).

45. DuPont Polyimide Films general information. Available at: http://www.dupont. com/content/dam/dupont/products-and-services/membranes-and-films/ polyimde-films/documents/DEC-Kapton-general-specs.pdf. Accessed $14 \mathrm{Mar}$ 2018.

46. Richardson, R. R., Miller, J. A. \& Reichert, W. M. Polyimides as biomaterials: preliminary biocompatibility testing. Biomaterials 14, 627-635 (1993).

47. Somasiri, N. L. D., Zenner, R. L. D., \& Houge, J. C. A process for surface texturing of Kapton polyimide to improve adhesion to metals. In IEEE Transactions on Components, Hybrids, and Manufacturing Technology, Vol. 14, no. 4, 798-801 (1991). https://doi.org/10.1109/33.105136

48. Gerardo, C. D., Cretu, E. \& Rohling, R. Fabrication of circuits on flexible substrates using conductive SU-8 for sensing applications. Sensors 17, 1420 (2017).

49. Koester, D., Cowen, A., Mahadevan, R., Stonefield, M. \& Hardy, B. PolyMUMPs design handbook. MEMSCAP Inc. (2003). Available at: http://www.memscap.
com/_data/assets/pdf_file/0019/1729/PolyMUMPs-DR-13-0.pdf. Accessed 3 Apr 2017.

50. Spicer, D., Crawford, J., Twanow, C., Wakefield, N., MicraGEM-Si ${ }^{\text {TM }}$, a flexible process platform for complex MEMS devices. Micralyne. Available at: https:// www.micralyne.com/wp-content/themes/micralyne/files/ resource micragem si.pdf. Accessed 3 Apr 2017.

51. Fthenakis, V. M. \& Moskovitz, P. D. An assessment of silane hazards. Solid State Technol. 33, 81-85 (1990).

52. Huang, Y., Hæggstrom, E. O., Zhuang, X., Ergun, A. S. \& Khuri-Yakub, B. T. A solution to the charging problems in capacitive micromachined ultrasonic transducers. Ultrason. Ferroelectr. Freq. Control IEEE Trans. 52, 578-580 (2005).

53. Park, K. K., Kupnik, M., Lee, H. J., Oralkan, Ö. \& Khuri-Yakub, B. T. Zero-bias resonant sensor with an oxide-nitride layer as charge trap. In 2010 IEEE Sensors 1024-1028 (2010). https://doi.org/10.1109/ICSENS.2010.5690742.

54. Wygant, I. O. et al. $50 \mathrm{kHz}$ capacitive micromachined ultrasonic transducers for generation of highly directional sound with parametric arrays. Ultrason. Ferroelectr. Freq. Control IEEE Trans. 56, 193-203 (2009).

55. Jensen, J. A., Nikolov, S. I., Gammelmark, K. L. \& Pedersen, M. H. Synthetic aperture ultrasound imaging. Ultrasonics 44, e5-e15 (2006).

56. Daft, C. M. W. Conformable transducers for large-volume, operatorindependent imaging. In 2010 IEEE International Ultrasonics Symposium 798-808 (San Diego, CA). https://doi.org/10.1109/ULTSYM.2010.5936021.

57. Singh, R. S. et al. in: Acoustical Imaging (eds Andre, M. P., Jones, J. P. \& Lee, H.) 211-222 (Springer, Netherlands, 2011).

58. Zhuang, X., Lin, D. S., Oralkan, Ö. \& Khuri-Yakub, B. T. Fabrication of flexible transducer arrays with through-wafer electrical interconnects based on trench refilling with PDMS. J. Microelectromech. Syst. 17, 446-452 (2008).

59. Chen, A. I. H. et al. Fabrication of a curved row \#x2013; column addressed capacitive micromachined ultrasonic transducer array. J. Microelectromech. Syst. 25, 675-682 (2016).

60. McLean, J. \& Degertekin, F. L. Interdigital capacitive micromachined ultrasonic transducers for sensing and pumping in microfluidic applications. In Transducers, Solid-State Sensors, Actuators and Microsystems, 12th International Conference, 915-918 (Boston, MA, 2003). https://doi.org/10.1109/ SENSOR.2003.1215624.

61. Yaralioglu, G. Ultrasonic heating and temperature measurement in microfluidic channels. Sens. Actuators Phys. 170, 1-7 (2011).

62. Joseph, J., Singh, S. G. \& Vanjari, S. R. K. A low pull-in SU-8 based capacitive micromachined ultrasonic transducer for medical imaging applications. In 2014 36th Annual International Conference of the IEEE Engineering in Medicine and Biology Society, 1398-1401 (Chicago, IL). https://doi.org/10.1109/ EMBC.2014.6943861.

63. Asaoka, Y. et al. 4.1: Distinguished paper: cavity shape control of the roll-to-roll fabricated novel microstructure film for improving the viewing-angle characteristics of LCDs. SID Symp. Dig. Tech. Pap. 45, 17-20 (2014). 